\title{
Processing Navon letters can make wines taste different
}

\author{
Michael B Lewis, Jennifer Seeley, Chris Miles
}

School of Psychology, Cardiff University, Park Place, Cardiff CF10 3AT, Wales, UK; e-mail: LewisMB@cf.ac.uk

Received 25 September 2008, in revised form 22 March 2009; published online 17 July 2009

\begin{abstract}
Previous work has demonstrated that providing a verbal description of a wine impairs its recognition (Melcher and Schooler, 1996 Journal of Memory and Language 35 231-245). It was proposed that the effect was due to disruption of the perceptual memory by the verbalisation process as seen in face recognition. A similar impairment can be observed in face recognition after reading the small (local) letters of Navon stimuli. Here, it is suggested that the effect in wine recognition is due to a change in the processing style following the provision of a verbal description. In the current experiment we investigated whether the reading of Navon letters also affected the recognition of wines. We found that wine recognition was more accurate after the reading of the global letters rather than after the reading of the local letters of the Navon stimuli. The results demonstrate how the control of visual processing can affect perception in other modalities.
\end{abstract}

\section{Introduction}

Perceptual memories, such as memories for faces, are difficult to describe verbally. Whilst we may recognise a person, it is difficult to describe verbally the recognition process. Indeed, it has been shown that the provision of a verbal description for the perceptual memory of an unfamiliar face results in poorer subsequent recognition of that face (Schooler and Engstler-Schooler 1990). This effect was labelled verbal overshadowing, on the assumption that the veridical perceptual memory is overshadowed (or dominated) by the abstracted verbal summary (see Chin and Schooler 2007, for a review).

Faces are not the only stimuli to exhibit such verbal overshadowing. For example, Melcher and Schooler (1996) demonstrated a similar effect for the recognition of wines. Participants were categorised as either novices, intermediates, or experts on the basis of their knowledge and experience with wines. In their experiment, a wine was tasted by the participant who then either did, or did not, provide a verbal description of the wine. For intermediate participants, those who described the wine were poorer at recognising it later from an array of four similar wines compared to those who did not describe it; wine experts and novices did not show this effect. This effect was attributed to the process of verbal overshadowing, where the perceptual memory of the wine is disrupted by committing the memory to words in a non-expert way. There was something about the content of the descriptions provided by the intermediates that was disrupting but was not present or was not disrupting in the descriptions provided by the experts and novices. The descriptions of the wines were analysed for content and some differences were observed, such as experts using more specialised terms.

Since Melcher and Schooler's (1996) wine experiment was conducted, our understanding of the verbal overshadowing effect for faces has changed. For example, a similar detriment in recognition is observed if the participant describes a face that is different from the target face (Dodson et al 1997) or even provides a description for an unrelated object such as a car (Brown and Lloyd-Jones 2003). The direct disruption of the perceptual memory by a verbal description is not consistent with these newer findings as the 
verbal description of a car could not overshadow the perceptual memory of a face. An alternative explanation was put forward, founded upon the idea of transfer-appropriate processing (Schooler et al 1997).

The transfer-appropriate processing account of the verbal overshadowing effect is based on the idea that faces are typically automatically processed in a holistic manner (see Tanaka and Farah 1993). When a face is seen during the learning phase, the assumption is that it is encoded in a holistic manner. That is, it is not encoded in terms of face-specific features (eg mouth shape, eye colour, etc). The process of verbally describing the face, or a different face, or a car, forces the participant to focus on stimulus-specific verbalisable features (ie to identify local rather than global features). Consequently, when the target face and distractor faces are presented at test, they are processed in the same feature-based manner carried over from their earlier description. Thus there is a mismatch between the recognition phase based on local features and the encoding phase based on global features. This mismatch between the encoding representation and the representation available at test leads to the observed poorer performance.

The same kind of transfer-appropriate processing account could be used to explain Melcher and Schooler's (1996) results in their wine experiment (such an explanation was put forward by Chin and Schooler 2007). Typically, wine drinkers may represent their perceptual experience of a wine in an automatic or holistic manner (that is the experience of the wine as a combination of various elements). Those participants who were required to describe the wine were directed towards an analytic or featural processing style (that is, the analysis and identification of the variety of elements that make up the whole experience) and this style persisted to the test phase. The shift from a holistic to a featural processing style might explain the poorer recognition in the discrimination task. The wine experts, who did not show the impairment, may have developed a featural processing style to all their wine tasting and so they would not show the same mismatch as non-experts. In support of this, Melcher and Schooler found that wine experts' descriptions differed from those of non-experts in that they had greater precision and were able to 'unpack' the broader characteristics of the wine.

In the face-recognition paradigm, the idea of a shift in processing style was further tested by removing the need for the provision of any verbal description to obtain the same effect. Macrae and H L Lewis (2002) used a letter-reading task to bias participants towards either a featural (or local) processing style or a holistic (or global) processing style. In an eyewitness line-up-style experiment, participants were presented with a series of Navon letters (see Navon 1977) between the encoding and the recognition phase. Navon letters are large (global) letters formed from a repeated smaller (local) letter (see figure 1). Participants who were required to read out the large letter performed better in the face-recognition phase compared to those who read out the small letters. This remarkable finding has been replicated (eg Hills and Lewis 2007a; Perfect et al 2007) and demonstrates that 5 min of Navon letter processing can disrupt the ability to recognise an earlier presented face.

Recently, Perfect et al (2008) demonstrated that the particular properties of the Navon stimuli were important in order to produce the effect. Typically, a Navon stimulus is generated such that the global letter is more obvious and so has precedence. Perfect and colleagues showed that by using Navon stimuli with a local precedence (ie with the local letter more obvious than the global letter) the effect on subsequent face recognition was reversed. It appears that it may be a requirement to inhibit the automatic response in favour of a controlled or analytic response that leads to the Navon effect of face recognition. Such an explanation also accounts for M B Lewis's (2006) finding that cryptic crosswords (but not standard crosswords) disrupt subsequent face recognition. 


\section{NNNNNNNNNNN}

NNNNNNNNNNNN

$\begin{array}{lr}\text { NNNN } & \text { NNNNN } \\ \text { NNNN } & \text { NNNN } \\ \text { NNNN } & \text { NNNN } \\ \text { NNNN } & \text { NNNN } \\ \text { NNNN } & \text { NNNN }\end{array}$

\section{NNNN NNNN}

NNNNNNNNNNN

\section{NNNNNNNNNNN}

NNNN NNNNN

NNNN NNNN

NNNN NNNN

NNNN NNNN

NNNN NNNN

NNNN NNNN

NNNN NNNN
Figure 1. Example of a Navon letter. A global 'R' is formed out of repeated smaller 'N's.

If the explanation for the verbal overshadowing effect observed in wine recognition is the same as that for faces, then this leads to the prediction that a 5 min Navon task will also affect the recognition of wines. Reading local Navon letters prior to a wine recognition test should impair later wine recognition compared to a condition where global Navon letters are read. In the experiment described here we tested this hypothesis.

\section{Method}

\subsection{Participants}

The participants were fifty Cardiff University undergraduates with an age range of 18 to 29 years (mean age, 19 years 7 months). The inclusion criterion for participation was that they were occasional or regular drinkers but were neither wine experts, nor had an alcohol-related drinking problem. Participants varied in their wine-drinking behaviour from drinking it 2 to 3 times a week to drinking it 2 to 3 times a year. ${ }^{(1)}$

\subsection{Materials}

A set of Navon letters were used as generated by Brand (2005). The stimuli had previously been employed in experiments that replicated the Navon effect in face recognition (Hills and M B Lewis 2007b; M B Lewis et al 2009). These were large letters formed from repeating a different smaller letter presented on a computer monitor such that their height was $100 \mathrm{~mm}$ and their width was $50 \mathrm{~mm}$. The monitor was viewed from a distance of approximately $0.5 \mathrm{~m}$.

A set of six different white wines was used for the wine tasting. These wines originated from six different countries and varied in their grape. In order to ensure a constant tasting experience across an experiment that lasted a few weeks, only wines that were available in boxes with tap dispensers were selected. The wines were presented in transparent plastic cups. Bread and water was provided to clear the palate between tasting of the wines. All the wine samples were hidden, unless they were being tasted at that time.

(1) Only one participant would have been in Melcher and Schooler's (1996) novice group whereas the remainder would have been in their intermediate group. Removal of the novice would not have changed the significance of the results. 


\subsection{Procedure}

Participants completed a questionnaire on their wine drinking behaviour and received basic instructions on the process of wine tasting and the format of the rest of the experiment. The experiment was conducted in two trials. In the first trial, the participant was presented with $25 \mathrm{ml}$ of a target wine to taste and given as much time as he/she wished. Immediately after tasting the wine, the participant was presented with a series of Navon letters via a computer monitor. The participant was required to read out either the global Navon letters or the local Navon letters (depending on which condition they were in). Each Navon letter was presented for $5 \mathrm{~s}$ and 60 letters were presented in total. Immediately after the Navon task, participants were presented with three wines for tasting. One wine was the target wine already tasted and two others were distractor wines. These were revealed to the participant simultaneously and the participants worked through the three wines in a self-paced manner until they made a decision. The participant tasted each of the wines in a predetermined counterbalanced order and selected which wine was the target wine. This concluded the first trial.

Following this first trial, the participant read an unrelated passage for 5 min before taking part in the second trial. The second trial took the same format as the first, except that it used three different wines and the Navon task was reversed (participants who did the global task in the first trial did the local task in the second trial and vice versa). Again, the participant attempted to identify the target wine from the three wines presented. A graphical depiction of the experiment is presented in figure 2 for clarification.

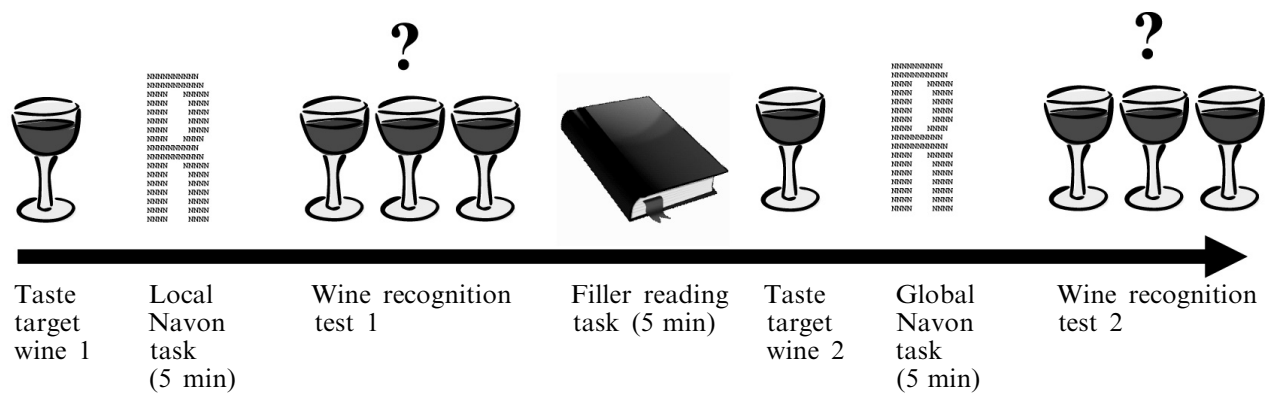

Figure 2. Graphical representation of the procedure for a participant in the local then global condition. The order of events runs from left to right.

\subsection{Design}

The independent variable was the nature of the Navon task (global or local) prior to the wine recognition test. This was a within-participants variable. The dependent variable was whether the participant correctly recognised the target wine in the recognition test. The order of the independent variable was counterbalanced across participants. The sets of three wines in the global and local conditions were also counterbalanced. Within the sets, the target wine was rotated between participants.

The design used only a single discrimination after each Navon task. This was because it was expected that any Navon effect would be shortlived. Hills and M B Lewis (2007b) demonstrated the rapidly decaying nature of the effect for face recognition, whereas Fallshore and Schooler (1995) had shown that the same was true for the verbal overshadowing effect.

\section{Results}

During the experiment, participants attempted to recognise a total of one hundred wines: fifty participants each performing two recognition tasks. Table 1 shows the results: over twice as many participants showed correct recognition following the global task 
Table 1. Pattern of data observed for the fifty participants. Participants were more accurate after performing the global Navon task than after performing the local Navon task.

\begin{tabular}{lll}
\hline Navon task & \multicolumn{2}{l}{ Recognition } \\
\cline { 2 - 3 } & correct & incorrect \\
\hline After global task & 32 & 18 \\
After local task & 23 & 27 \\
\hline
\end{tabular}

but not the local task $(n=16)$ than showed the reverse pattern $(n=7)$. The data were analysed with a one-way sign test. ${ }^{(2)}$ This shows that performance was significantly better $(p<0.05)$ after the global task than after the local task.

Similar analysis of only the first trial or second trial for each participant led to a similar pattern overall, but neither reached significance on its own. There was no evidence for an attenuation of the Navon effect after the first trial as shown by Fallshore and Schooler (1995) for verbal overshadowing.

\section{Discussion}

The finding supports the suggestion that global Navon processing leads to better performance $(64 \%$ accuracy) in a later wine recognition task than local Navon processing (46\% accuracy). There are at least two consequences of this result.

First, with regard to Melcher and Schooler's (1996) finding of a verbal overshadowing effect in wine recognition, it appears that their effect is not caused by the description of the wine. If we translate Perfect and colleagues' (2008) controlled processing account of the Navon effect to the current experiment then it would appear that the controlled and analytic responding required to do the local Navon task carries over into the winerecognition task. This carry-over impacts upon the performance of participants presented with the three wines making recognition poorer than after the global Navon task. As there is no control condition it may also be that the global task makes participants more automatic in their responding following the global Navon task and hence there is improved performance in wine recognition than when following the local Navon task.

In Melcher and Schooler's (1996) experiment, the verbal overshadowing effect was only found for intermediate wine drinks. The current findings offer a possible explanation for this. The novice wine drinkers may not have the ability to provide verbal descriptions that were sufficiently analytic in order to establish a change in style that could carry over to the wine-recognition stage. The experts, on the other hand, may have been analytic in their approach to the initial wine tasting and so, even if the verbal description task did produce a carry-over of a controlled analytic style, then this style would not have been deleterious to their wine recognition skills.

Second, the result suggests that the Navon effect observed for face can cross modalities. Wine discrimination is primarily an olfactory process (although we cannot discount the possibility that the discriminations were based on colour or texture), whereas the Navon task is clearly a visual task. Explanations of the Navon effect that are based on simple visual properties (such as spatial frequencies) are too limited. The processing of Navon stimuli appears to change the way we perceive the world in a far more general way than merely our visual processing (albeit for a limited time).

The change from automatic to controlled responding, as put forward by Perfect and colleagues (2008), is an explanation that allows the effect to cross modalities. Faces, wine, and possibly many other stimuli are typically processed in an automatic and fast manner. This fast analysis would be efficient and memories would be stored

(2) For those who are comfortable with parametric tests, a paired one-tailed $t$-test was also performed. This test, too, showed a significant effect $(t=1.927, p=0.0299)$. 
in terms of this automatic analysis. The processing of the local letters in typical Navon stimuli will require controlled responses and this will carry over for a short time to whatever task is done immediately afterwards. If this task is face recognition then the face will be encoded in a controlled way. If it is wine tasting, then the wine will be encoded in a controlled way. Either way, the resulting encoded stimuli will be different in form than an automatically encoded stimulus and hence, according to this account, recognition would be poorer owing to a mismatch of memories. If, however, the stimulus was learnt and tested in the same controlled analytic way, then this mismatch would not occur (as demonstrated for faces by M B Lewis et al 2009).

The current research focused upon wine tasting, partly because there is evidence of the verbal overshadowing effect in that domain and partly because of an increasing interest in the perception of wine (see Gregory 2007). One would predict from the current results and the discussion that follows that a Navon effect would be found for any task where it is possible to encode the items in an analytic manner but where the default would be to process them in an automatic way.

\section{References}

Brand A, 2005 Explaining the Verbal Overshadowing Effect unpublished $\mathrm{PhD}$ thesis, Cardiff University, Cardiff, UK

Brown C, Lloyd-Jones T J, 2003 "Verbal overshadowing of multiple face and car recognition: Effects of within- versus across-category verbal descriptions" Applied Cognitive Psychology 17 $183-201$

Chin J M, Schooler J W, 2007 "WWhy do words hurt? Content, process, and criterion shirt accounts of verbal overshadowing" European Journal of Cognitive Psychology 20 396-413

Dodson C S, Johnson M K, Schooler J W, 1997 "The verbal overshadowing effect: why descriptions impair face recognition" Memory \& Cognition 25129 - 139

Fallshore M, Schooler J W, 1995 "Verbal vulnerability of perceptual expertise" Journal of Experimental Psychology: Learning, Memory, and Cognition 21 1608-1623

Gregory R L, 2007 "Wine tasting” Perception 36321 - 322

Hills P J, Lewis M B, 2007a "Testing alternatives to Navon letters to induce transfer-inappropriate processing shift in face recognition" European Journal of Cognitive Psychology $20561-576$

Hills P J, Lewis M B, 2007b "Temporal limitation of Navon effect of face recognition" Perceptual and Motor Skills $104501-509$

Lewis M B, 2006 "Eye-witnesses should not do cryptic crosswords prior to identity parades" Perception $351433-1436$

Lewis M B, Mills C, Hills P J, Weston N, 2009 "Navon letters affect face learning and face retrieval" Experimental Psychology 56258 - 264

Macrae C N, Lewis H L, 2002 "Do I know you? Processing orientation and face recognition" Psychological Science $13194-196$

Melcher J M, Schooler J W, 1996 "The misremembrance of wines past: verbal and perceptual expertise differentially mediate verbal overshadowing of taste memory" Journal of Memory and Language $35231-245$

Navon D, 1977 "Forest before the trees: The precedence of global features in visual perception" Cognitive Psychology $9353-383$

Perfect T J, Dennis I, Snell A, 2007 "The effect of local and global processing bias on eyewitness identification performance" Memory $15784-798$

Perfect T J, Weston N J, Dennis I, Snell A, 2008 "The effects of precedence on Navon-induced processing bias in face recognition" Quarterly Journal of Experimental Psychology 61 1479-1486

Schooler J W, Engstler-Schooler T Y, 1990 "Verbal overshadowing of visual memories: some things are better left unsaid" Cognitive Psychology 17 36-71

Schooler J W, Fiore S M, Brandimonte M A, 1997 "At a loss from words: verbal overshadowing of perceptual memories", in The Psychology of Learning and Motivation Ed. D L Medin (San Diego, CA: Academic Press) pp $291-340$

Tanaka J R, Farah M J, 1993 "Parts and wholes in face recognition" Quarterly Journal of Experimental Psychology 46 225-245 


\section{PERTEPTION}

VOLUME 382009

www.perceptionweb.com

Conditions of use. This article may be downloaded from the Perception website for personal research by members of subscribing organisations. Authors are entitled to distribute their own article (in printed form or by e-mail) to up to 50 people. This PDF may not be placed on any website (or other online distribution system) without permission of the publisher. 